5 Strugnell WE, Slaughter IR, Riley RA, et al. Modified RV short axis series - a new method for cardiac MRI measurement of right ventricular volumes. J Cardiovasc Magn Reson 2005; 7: 769-774.

6 Sanz J, Garcia-Alvarez A, Fernandez-Friera L, et al. Right ventriculo-arterial coupling in pulmonary hypertension: a magnetic resonance study. Heart 2012; 98: 238-243.

7 D'Alto M, Ghio S, D'Andrea A, et al. Inappropriate exercise-induced increase in pulmonary artery pressure in patients with systemic sclerosis. Heart 2011; 97: 112-117.

8 Champion HC, Michelakis ED, Hassoun PM. Comprehensive invasive and noninvasive approach to the right ventricle-pulmonary circulation unit: state of the art and clinical and research implications. Circulation 2009; 120: 992-1007.

9 Vonk-Noordegraaf A, Haddad F, Chin KM, et al. Right heart adaptation to pulmonary arterial hypertension: physiology and pathobiology. J Am Coll Cardiol 2013; 62: Suppl 25, D22-D33.

10 Sagawa K, Maughan L, Suga H, et al. Cardiac contraction and the pressure-volume relationship. New York, Oxford University Press, 1988.

11 Tedford RJ, Mudd JO, Girgis RE, et al. Right ventricular dysfunction in systemic sclerosis-associated pulmonary arterial hypertension. Circ Heart Fail 2013; 6: 953-963.

12 Woods PR, Frantz RP, Taylor BJ, et al. The usefulness of submaximal exercise gas exchange to define pulmonary arterial hypertension. J Heart Lung Transplant 2011; 30: 1133-1142.

\title{
Shorter treatment for multidrug-resistant tuberculosis: the good, the bad and the ugly
}

\author{
To the Editor:
}

We welcome the initiative by the Guideline Development Group (GDG) members to issue the 2016 update of World Health Organization (WHO) treatment guidelines for drug-resistant tuberculosis (TB) [1]. With one in two patients currently failing on treatment for multidrug-resistant (MDR)-TB, primarily as a result of the difficulties presented by cumulative drug toxicity, logistics, costs and subsequent poor adherence to therapy [2], a shorter regimen for selected patients would be a tremendous asset, even though the GDG argues that the recommendation is conditional, and the scientific evidence for the recommendation is low. Since the first reports on the efficacy of a regimen of only 9 months for MDR-TB [3, 4], two more studies have been published to support the concept of shorter regimens $[5,6]$ while the STREAM (Evaluation of a Standard Treatment Regimen of Anti-tuberculosis Drugs for Patients with MDR-TB) study is still enrolling [7]. However, shortening therapy would only apply for selected patients without prior use of or proven resistance to fluoroquinolones (group A) or second-line injectable agents (group B). At least five active drugs should be available for the intensive phase (4-6 months). Further exclusions are extrapulmonary TB, additional resistance to pyrazinamide (PZA) and pregnancy. Clofazimine [8] and linezolid [9] were regrouped as core agents in group $C$ with ethionamide or prothionamide, while para-aminosalicylic acid was deferred to group D.

The GDG noted the lack of evidence for shortening treatment for extrapulmonary MDR-TB and did not recommend doing so, even though drug-susceptible extrapulmonary and pulmonary $\mathrm{TB}$ are treated with similar schedules and durations of treatment. MDR-TB treatment requires bacteriological monitoring, which is more feasible in sputum samples from pulmonary MDR-TB patients than samples from extrapulmonary MDR-TB cases.

Excluding PZA resistance is problematic. PZA susceptibility testing is difficult and not widely available, especially in low-resource settings. However, recent studies have shown excellent concordance of molecular and phenotypic susceptibility $[10,11]$, and testing molecular susceptibility to this important drug may become an important asset, even in low-resource settings.

We retrospectively analysed how many MDR-TB patients in the Netherlands would potentially have benefitted from the new guidelines; we therefore studied the data of all 172 consecutive patients that had started treatment since 2000. Data on patients treated between 2000 and 2009 have been published previously [8].

Five of these 172 patients had earlier MDR-TB treatment, four were pregnant, 30 had extrapulmonary MDR-TB, 28 had additional resistance to group A and/or group B drugs and 52 had PZA-resistant organisms. Four had extensively drug-resistant TB (these patients were included in our earlier report [8]), and all of these cases had a favourable outcome. As multiple exclusion criteria clustered in some patients 
(table 1), nearly half (85 (49.4\%) out of 172$)$ of our patients would meet the criteria for the new shorter regimen. Interestingly, $58 \%$ of the $2000-2009$ cohort, but only $36 \%$ (25 out of 69 ) of the $2010-2015$ cohort would be eligible for shortened treatment. Of the 54 patients starting treatment between 2010 and 2013, 46 (85.2\%) completed treatment successfully, one died from an unrelated cause, four interrupted their treatment and three left the Netherlands to complete their treatment elsewhere. Therefore, the high success rate at $\sim 85 \%$ reported earlier [8], without failure or relapse was maintained.

The evidentiary table on page 20 in the guidelines [1] is based on data from 1205 patients; 89 of those were lost to follow-up. Outcome in those without resistance to quinolones, injectables or PZA was successful in 121 (96.8\%) out of 125, which is obviously excellent, but the lower limit of the confidence interval (77.3-99.6\%) calls for caution.

MDR-TB treatment outcome in the Netherlands with conventional treatment of 20 months duration $[8,12]$ has been highly successful with very limited toxicity, and no failure or relapse. We benefitted from an extraordinary collaboration between tertiary treatment centres and public health physicians, with hardly any loss to follow-up. We have treated patients in whom treatment was extremely long, considering the fact that their lesions and their bacterial load were limited. Whether treatment duration is important in cavitary lesions and in patients with a large initial bacterial load needs to be investigated in future studies.

We believe that part of our success is due to an approach using tailored pharmacokinetic/ pharmacodynamic (PK/PD) modelling. Drug susceptibility testing in MDR-TB is important [13], but merely reporting a test result below or above the European Committee on Antimicrobial Susceptibility Testing breakpoint may not provide sufficient precision. Clearly, the contribution of each individual drug depends on drug exposure relative to the drug susceptibility of the organism [14]. Molecular tests such as the MTBDRplus (version 2.0; Hain Lifescience, Nehren, Germany) to predict susceptibility to second-line drugs currently do not provide a minimum inhibitory concentration (MIC) value to allow for PK/PD calculations, while MIC values for second-line drugs have gradually changed over time. All of these considerations imply that vigilance is warranted with shortened treatment. Modern diagnostic tools should enable physicians to act promptly if treatment is stalling [15]. We believe that drug concentration monitoring has added value to fast molecular tests and drug susceptibility testing to support the shortened regimen by preventing slow response due to low drug exposure [16]. Synergy between second-line TB drugs may further benefit the short course of treatment [17].

We welcome shortened treatment and we agree that the good news of shortened treatment duration should be carefully monitored. Only four of our eligible patients were still sputum smear microscopy positive after 4 months of treatment; 81 (95\%) would therefore have continued treatment for 9 months only, according to the new guideline; for these 81 patients, 9 months of treatment would remain good news during the planned 9 months of treatment, while only four would have had to extend their treatment to 12 months according to the new guidelines. However, if many more patients among the 85 starting the 9- (or 12-) month regimen would relapse because of failure to sterilise persister organisms, their treatment would become a negative experience, with a treatment duration of 9 months plus an additional 20 months. Both for low-resource settings and for programmes that currently have excellent treatment results, monitoring is therefore essential to avoid the bad and ugly effects of what is intended to be simply good.

TABLE 1 Reasons for ineligibility of 172 multidrug-resistant tuberculosis (MDR-TB) patients in the Netherlands to receive treatment of a shortened duration (9-12 months) rather than regular care (18-20 months), 2000-2015

\begin{tabular}{|c|c|c|c|c|}
\hline $\begin{array}{c}\text { Previous } \\
\text { MDR-TB drugs }\end{array}$ & Pregnancy & $\begin{array}{c}\text { Extrapulmonary } \\
\text { TB }\end{array}$ & $\begin{array}{l}\text { Resistance to FLQ\# } \\
\text { or second-line } \\
\text { injectable drugs }\end{array}$ & PZA resistance \\
\hline
\end{tabular}

\begin{tabular}{|c|c|c|c|c|c|c|}
\hline Previous MDR-TB drugs & 5 & 0 & 0 & 4 & 3 & \\
\hline Pregnancy & & 4 & 2 & 0 & 0 & \\
\hline Extrapulmonary TB & & & 28 & 2 & 9 & \\
\hline $\begin{array}{l}\text { Resistance to } \mathrm{FLQ}^{\#} \text { or second-line } \\
\text { injectable drugs }\end{array}$ & & & & 22 & 12 & \\
\hline PZA resistance & & & & & 28 & \\
\hline Patients meeting exclusion criterion & 5 & 4 & 30 & 28 & 52 & 87 \\
\hline
\end{tabular}


Richard van Altena ${ }^{1}$, Onno W. Akkerman ${ }^{1}$, Jan-Willem C. Alffenaar ${ }^{2}$, Huib A.M. Kerstjens ${ }^{1}$, Cecile Magis-Escurra ${ }^{3}$, Martin J. Boeree ${ }^{3}$, Dick van Soolingen ${ }^{4,5}$, Wiel C.M. de Lange ${ }^{1}$, Mathieu S. Bolhuis ${ }^{2}$, Wouter Hoefsloot ${ }^{3}$, Gerard de Vries $^{6,7}$ and Tjip S. van der Werf ${ }^{1,8}$

${ }^{1}$ University of Groningen, University Medical Center Groningen, Dept of Pulmonary Diseases and Tuberculosis, Groningen, The Netherlands. ${ }^{2}$ University of Groningen, University Medical Center Groningen, Dept of Clinical Pharmacy and Pharmacology, Groningen, The Netherlands. ${ }^{3}$ Tuberculosis Center, University Center for Chronic Diseases Dekkerswald, Radboud Nijmegen University Medical Center, Nijmegen, The Netherlands. ${ }^{4}$ Tuberculosis Reference Laboratory, Centre for Infectious Diseases, National Institute for Health and the Environment (RIVM), Bilthoven, The Netherlands. ${ }^{5}$ Depts of Medical Microbiology and Lung Disease, Radboud Nijmegen University Medical Center, Nijmegen, The Netherlands. ${ }^{6} \mathrm{KNCV}$ Tuberculosis Foundation, The Hague, The Netherlands. ${ }^{7}$ Centre for Infectious Diseases, National Institute of Public Health and the Environment, Bilthoven, The Netherlands. ${ }^{8}$ University of Groningen, University Medical Center Groningen, Dept of Internal Medicine, Groningen, The Netherlands.

Correspondence: Tjip S. van der Werf, University of Groningen, University Medical Center Groningen, Dept of Pulmonary Diseases and Tuberculosis, and Dept of Internal Medicine, Infectious Diseases, Groningen, PO Box 30.001, 9700 RB Groningen, The Netherlands. E-mail: t.s.van.der.werf@umcg.nl

Received: June 192016 | Accepted after revision: Aug 222016 | First published online: Oct 062016

Conflict of interest: Disclosures can be found alongside this article at erj.ersjournals.com

\section{References}

1 World Health Organization (WHO). WHO Treatment Guidelines for Drug-resistant Tuberculosis. 2016 Update. Geneva, WHO, 2016.

2 Johnston JC, Shahidi NC, Sadatsafavi M, et al. Treatment outcomes of multidrug-resistant tuberculosis: a systematic review and meta-analysis. PLoS One 2009; 4: e6914.

3 Van Deun A, Maug AKJ, Salim MAH, et al. Short, highly effective, and inexpensive standardized treatment of multidrug-resistant tuberculosis. Am J Respir Crit Care Med 2010; 182: 684-692.

4 Aung KJM, Van Deun A, Declercq E, et al. Successful "9-month Bangladesh regimen" for multidrug-resistant tuberculosis among over 500 consecutive patients. Int J Tuberc Lung Dis 2014; 18: 1180-1187.

5 Piubello A, Harouna SH, Souleymane MB, et al. High cure rate with standardised short-course multidrug-resistant tuberculosis treatment in Niger: no relapses. Int J Tuberc Lung Dis 2014; 18: 1188-1194.

6 Kuaban C, Noeske J, Rieder HL, et al. High effectiveness of a 12-month regimen for MDR-TB patients in Cameroon. Int J Tuberc Lung Dis 2015; 19: 517-524.

7 Nunn AJ, Rusen ID, Van Deun A, et al. Evaluation of a standardized treatment regimen of anti-tuberculosis drugs for patients with multi-drug-resistant tuberculosis (STREAM): study protocol for a randomized controlled trial. Trials 2014; 15: 353.

8 van Altena R, de Vries G, Haar CH, et al. Highly successful treatment outcome of multidrug-resistant tuberculosis in the Netherlands, 2000-2009. Int J Tuberc Lung Dis 2015; 19: 406-412.

9 Bolhuis MS, Tiberi S, Sotgiu G, et al. Linezolid tolerability in multidrug-resistant tuberculosis: a retrospective study. Eur Respir J 2015; 46: 1205-1207.

10 Simons SO, van der Laan T, Mulder A, et al. Rapid diagnosis of pyrazinamide-resistant multidrug-resistant tuberculosis using a molecular-based diagnostic algorithm. Clin Microbiol Infect 2014; 20: 1015-1020.

11 Cambau E, Viveiros M, Machado D, et al. Revisiting susceptibility testing in MDR-TB by a standardized quantitative phenotypic assessment in a European multicentre study. J Antimicrob Chemother 2015; 70: 686-696.

12 Geerligs WA, van Altena R, De Lange WCM, et al. Multidrug-resistant tuberculosis: long-term treatment outcome in the Netherlands. Int J Tuberc Lung Dis 2000; 4: 758-764.

13 Bastos ML, Hussain H, Weyer K, et al. Treatment outcomes of patients with multidrug-resistant and extensively drug-resistant tuberculosis according to drug susceptibility testing to first- and second-line drugs: an individual patient data meta-analysis. Clin Infect Dis 2014; 59: 1364-1374.

14 Alffenaar JWC, Akkerman OW, Bolhuis MS, et al. Breakpoints and drug exposure are inevitably closely linked. Antimicrob Agents Chemother 2015; 59: 1384.

15 Ghimire S, Bolhuis MS, Sturkenboom MGG, et al. Incorporating therapeutic drug monitoring into the World Health Organization hierarchy of tuberculosis diagnostics. Eur Respir J 2016; 47: 1867-1869.

16 van der Burgt EPM, Sturkenboom MGG, Bolhuis MS, et al. End TB with precision treatment! Eur Respir J 2016; 47: 680-682.

17 Bolhuis MS, van der Laan T, Kosterink JGW, et al. In vitro synergy between linezolid and clarithromycin against Mycobacterium tuberculosis. Eur Respir J 2014; 44: 808-811. 